\title{
VCSEL with finite-size high-contrast metastructure
}

\section{Anjin Liu, Wanhua Zheng, Dieter Bimberg}

Anjin Liu, Wanhua Zheng, Dieter Bimberg, "VCSEL with finite-size highcontrast metastructure," Proc. SPIE 10812, Semiconductor Lasers and Applications VIII, 1081202 (6 November 2018); doi: 10.1117/12.2501231 


\title{
VCSEL with finite-size high-contrast metastructure
}

\author{
Anjin Liu ${ }^{\text {a,b,c }}$, Wanhua Zheng ${ }^{\mathrm{a}, \mathrm{b}, \mathrm{c}}$, and Dieter Bimberg ${ }^{\mathrm{d}, \mathrm{e}}$ \\ ${ }^{a}$ Laboratory of Solid-State Optoelectronics Information Technology, Institute of Semiconductors, \\ Chinese Academy of Sciences, Beijing, China \\ ${ }^{\mathrm{b}}$ State Key Laboratory on Integrated Optoelectronics, Institute of Semiconductors, Chinese Academy \\ of Sciences, Beijing, China \\ ${ }^{\mathrm{c} C}$ College of Materials Science and Opto-Electronic Technology, University of Chinese Academy of \\ Sciences, Beijing, China \\ ${ }^{\mathrm{d}}$ Institute of Solid State Physics and Center of Nanophotonics, Technische Universität Berlin, \\ Hardenbergstrasse 36, 10623 Berlin, Federal Republic of Germany \\ ${ }^{\mathrm{e}}$ Bimberg Chinese-German Center for Green Photonics of the Chinese Academy of Sciences at \\ CIOMP, Changchun, China
}

\begin{abstract}
High-contrast metastructures like one-dimensional high-contrast gratings (HCGs) are promising to improve the performance of conventional VCSELs, also presenting a basis for new applications. Different from the previous studies where HCGs are always modelled being of infinite size, we studied here the finite-size HCGs, which match the real situation. We observe finite-size HCGs behaving very differently from infinite-size HCGs. The reflectivity of a finitesize HCG strongly depends on the HCG size and the source size. At the same time, the simulation results show, that finite-size HCGs can shape the output beam, and a Gaussian-like reflected wave is typically achieved. Most important the normally incident light is partly redirected to the in-plane direction, showing unidirectional transmission. Monolithically integrated HCG-based optical sensors can be based on this novel effect. An integrable HCG reflector was fabricated with GaInP as the sacrificial layer targeting the application of HCG-VCSEL at $980 \mathrm{~nm}$ range. The measured reflectivity agrees well with the calculated reflectivity.
\end{abstract}

Keywords: Vertical-cavity surface-emitting lasers, subwavelength structure, mirrors, high-contrast grating, guided mode, finite size, optical sensor.

\section{INTRODUCTION}

Vertical-cavity surface-emitting lasers (VCSELs) have been important light sources for data communication (Datacom), computer communication (Computercom), sensing, consumer electronics, and much more, because they provide many notable advantages, e.g., the high modulation bandwidth, the low power consumption, the symmetric beam, and the ability of two-dimensional (2D) VCSEL array configuration. High-speed, energy-efficient, and high-temperature VCSELs for Datacom and Computercom with increasing transmission distance have been progressing rapidly in the last few years [1-6]. The application of VCSELs in smartphones for 3D sensing opens a door for the huge market of sensors in our daily life. VCSELs have been receiving increasing attention from industry and academic communities.

Recently nanoscale metastructures like one-dimensional high contrast gratings (HCGs) have been proposed and demonstrated unique optical properties [7]. A HCG is a subwavelength grating with grating bars composed of the high-index material fully surrounded by a low-index medium like the air, resulting in a high index contrast [8]. The grating period is in the near-wavelength regime, between the wavelength in the high-index material and the wavelength in the low-index material. Theoretical analysis shows the first two waveguide array modes with real propagation constants in infinite-size HCGs have a $\pi$-phase difference at the output plane and cancel each other causing a nearly $100 \%$ reflection. When two such $100 \%$ reflectivity points are located closely in the spectrum, a high-reflectivity broadband is obtained. There are alternative interpretations for the observed high-reflectivity broad band of HCGs based on the Fano resonance or guided-mode resonance [9,10]. A broadband and high-reflectivity HCG can serve as a reflector and replace a part of or fully the top DBR [11-13].

*liuanjin@semi.ac.cn

Semiconductor Lasers and Applications VIII, edited by Ning Hua Zhu, Werner H. Hofmann, Proc. of SPIE Vol. 10812, 1081202 · C 2018 SPIE · CCC code: 0277-786X/18/\$18 · doi: 10.1117/12.2501231 
Experimentally, HCG-VCSELs show an excellent mode selectivity and polarization control even for large oxide apertures.

In previous work, HCGs were always modelled as being of infinite size with an infinite-size incident plane wave. Only recently finite-size HCGs with a finite-size incident wave, being much closer to realistic cases of HCG-VCSELs, have been investigated $[14,15]$. Quite different properties of finite-size HCGs have been discovered. The finite size of the HCG reduces the bandwidth of high reflectivity spectra and higher-order angular components can excite the eigenmodes of the HCG. These eigenmodes reduce the reflectivity and enhances the transmittance of the HCG, and at the same time redirect the normally incident light to the in-plane direction showing unidirectional transmission, which is useful to construct monolithically integrated HCG-based optical sensors [16,17]. Also finite-size HCGs shaping the output beam for a Gaussian incident wave and a Gaussian-like reflected wave predicted in 2014 [14], has been experimentally confirmed recently [18].

\section{SIMULATION MODEL}

The HCG model studied here is shown in Fig. 1. Next to a HCG section with several bars, two slabs (i.e. waveguides) are at each side. HCG bars and slabs with high refractive index $\left(n_{\mathrm{H}}=3.2\right)$ are fully surrounded by a low-index $\left(n_{\mathrm{L}}\right)$ material (e.g. air). The finite-size Gaussian wave is at normal incidence to the HCG section. The HCG parameters are: $\Lambda=0.38 \mu \mathrm{m}, t=0.235 \mu \mathrm{m}$, and $a=0.25 \mu \mathrm{m}$ or $0.26 \mu \mathrm{m}$. The transverse magnetic (TM) polarization, electric component perpendicular to the HCG bars, is considered.

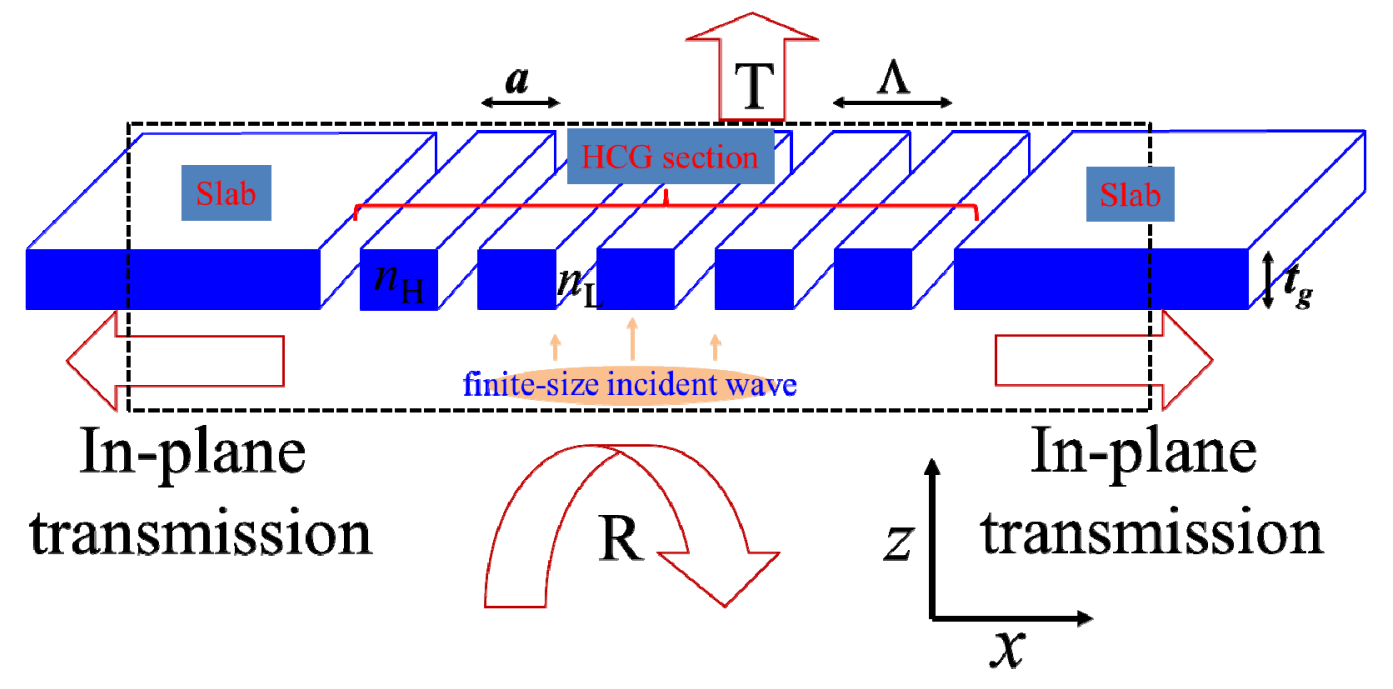

Fig. 1. Simulation model of a finite-size HCG; $a$ is the width of HCG bar; and $t_{g}$ is the thickness of HCG; $T$ and $R$ denote transmission and reflection, respectively; $n_{\mathrm{H}}$ is the refractive index of the bars; $n_{\mathrm{L}}$ is the refractive index of the medium surrounding the bars. The dashed rectangle represents the calculation domain with perfectly matched layers (PMLs) boundaries.

For the finite-size HCG, the two-dimensional finite-difference time-domain (2D FDTD) method is adopted to calculate the reflectivity spectrum with a pulse of a Gaussian source. The finite-size Gaussian source is placed at the center of HCG. The reflected and the transmitted waves are monitored. The reflectivity and transmittance here are defined by the ratios of the reflected power and transmitted power to the launched power, respectively. Perfectly matched layer (PML) boundary conditions are employed to describe the finite-size HCG. Also symmetric boundary condition in the $x$ direction can adopted to reduce the computational time and resource [14].

\section{EIGENMODES OF A HCG}

The fast Fourier transform is performed in the FDTD calculation of the HCG. Figure 2(a) shows the eigenmode spectrum of the HCG. Two peaks, corresponding to two eigenmodes, are shown here. Actually, there are other eigenmodes at the shorter wavelength side being of no importance for this study [17]. Modes 1 and 2 have wavelengths of 763.3 and 587 
$\mathrm{nm}$, respectively. The field distributions of the two modes (see Figs. 2(b) and (c)) have an odd symmetry with respect to the plane along the $z$ direction. Thus these two eigenmodes can't be excited by a plane wave which has only zero-order angular component, because the coupling coefficient between the incident plane wave and the eigenmode is zero. However, when the incident wave is of finite size, there are lots of higher-order angular components in the finite-size incident wave. The higher-order angular components can excite the eigenmodes, because the coupling coefficient between the finite-size incident wave and the eigenmode is nonzero. The coupling between the finite-size incident wave and the eigenmodes causes some unique phenomena, e.g., unidirectional transmission.

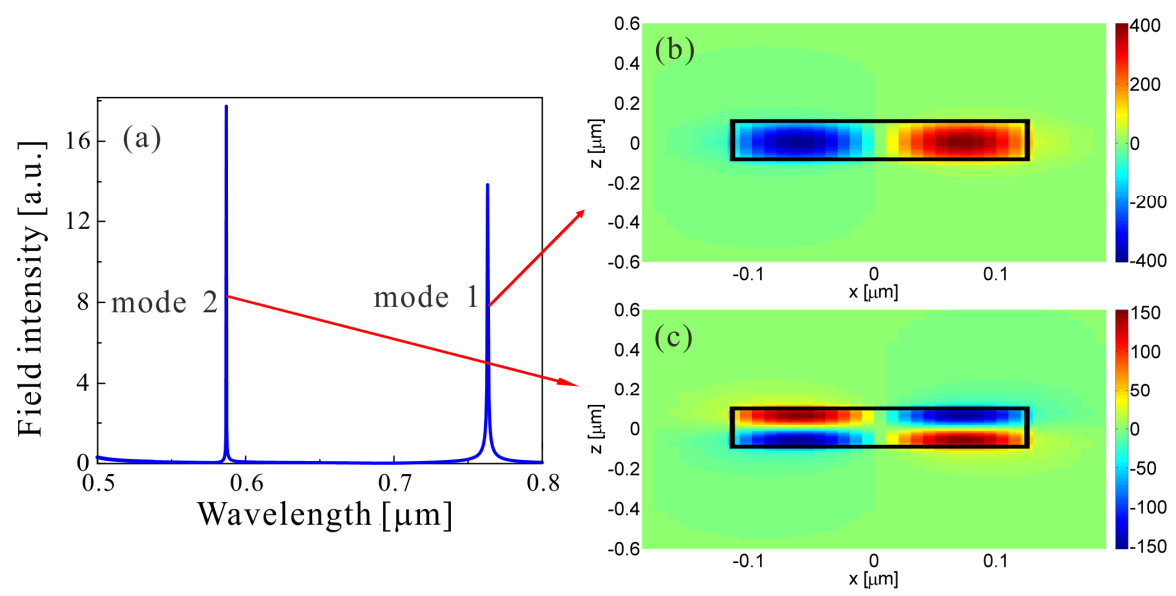

Fig. 2. (a) Eigenmode spectrum of the HCG. (b) Field distribution of mode 1 ( $H_{y}$ component). (c) Field distribution of mode 2 ( $H_{y}$ component). The HCG parameters are: $\Lambda=0.38 \mu \mathrm{m}, t=0.235 \mu \mathrm{m}$, and $a=0.26 \mu \mathrm{m}$.

\section{REFLECTIVITY AND TRANSMITTANCE OF A FINITE-SIZE HCG}

To study the effect of the HCG size, the excited source size (i.e. oxide aperture in the HCG-VCSEL) is fixed, and the HCG size is varied. Figure 3(a) shows the reflectivity spectrum for different HCG sizes under normal incidence. Compared with the slab without grating structure, the HCG greatly enhances the reflectivity around $850 \mathrm{~nm}$. However, the finite-size HCG has a smaller band of reflectivity larger than $99.5 \%$ and the reflectivity decreases compared to the infinite-size HCG [14]. The reflectivity increases with increasing HCG size. Interestingly, a dip occurs at $744.7 \mathrm{~nm}$ corresponding to the wavelength of the eigenmode, in the reflectivity spectrum independent of the HCG size. This dip and its position are independent of HCG size, while it disappears in the reflectivity spectrum of the infinite-size HCG. The transmission as shown in Fig. 3(b) decreases with increasing HCG size. Transmission shows a maximum at 744.7 nm, same as the dip in the reflectivity spectra in Fig. 3(a).

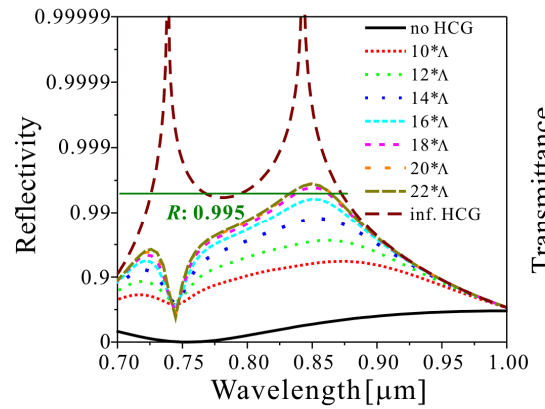

(a)

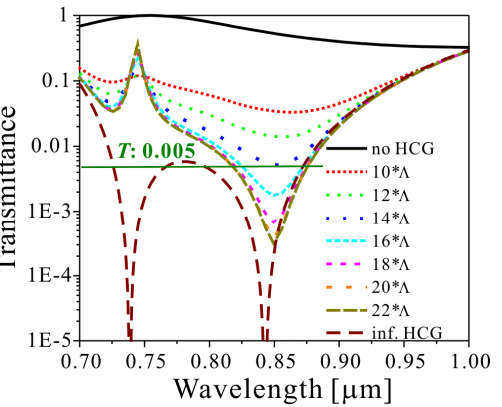

(b)

Fig. 3. Reflectivity spectra (a) and transmission spectra (b) for different HCG sizes with a fixed-size (4 $\mu$ m, $1 / e$ width of the Gaussian source) Gaussian source under normal incidence. The slab without grating structure and the infinite-size HCG are shown for comparisons. $\Lambda=0.38 \mu \mathrm{m}, t=0.235 \mu \mathrm{m}, a=0.25 \mu \mathrm{m}$. The horizontal lines indicate $99.5 \%$ reflectivity (a) and $0.5 \%$ transmission (b).

Figure 3 demonstrates some of the unique properties of finite-size HCGs, which do not exist for infinite-size HCGs. Comparing the reflectivity spectrum of the finite-size HCG with the infinite-size HCG under normal incidence, it is 
obvious that the HCG size greatly affects the reflectivity spectrum. For an infinite-size HCG, the incident wave is an infinite plane wave. There exists only the zero angular component in the angular spectrum. However, when the finitesize Gaussian source is incident on the finite-size HCG, there are many nonzero angular components in the angular spectrum of the Gaussian source. These nonzero angular components have the same effect as the off-normal incidence in the infinite-size HCG [14]. The nonzero angular components (i.e. higher-order angular components) can excite the eigenmodes, causing the appearance of the dip in the reflectivity spectrum resulting in a reduction of the high-reflectivity band width. On the other hand, the nonzero angular components in the finite-size source are beneficial for the mode selectivity in HCG-VCSELs.

\section{BEAM SHAPING}

Field profiles of the reflected waves for different HCG sizes are shown in Fig. 4(a). The reflected waves are Gaussianlike, because of the zero-order diffraction in the finite-size HCG. Thus, the reflected waves interact strongly with the gain medium in HCG-VCSELs thanks to the Gaussian-like reflected wave. A threshold current of below mA can be realized [11]. This means that finite-size HCGs can well confine the optical wave in a HCG-VCSEL cavity. As the finite-size HCG has losses into the slab and scattering at the edges occurs, the reflected and the transmitted field are no longer tightly connected. The output beam profiles affect coupling to the fiber and consequently high-speed link performance. Such far fields are shown for different configurations in Figs. 4(b) and (c). Figure 4(b) shows the profiles for different HCG sizes with a fixed-size source. There is a main lobe located in the center of the far field, and depending on the dimensions, several side lobes surrounding the main lobe can occur. The intensity of the side lobes decreases with increasing the HCG size. Figure 4(c) shows the far field profiles for a quite large HCG for different source sizes. Again, the far-field can vary from a well-focused Gaussian-like beam to a quite distorted far field. The intensity of the side lobes are best suppressed for a source size of $7 \mu \mathrm{m}$. From Fig. 4(c), we can find that the optimum source size for a fixed HCG size. This result shows that a comprehensive modelling as presented here is definitely needed to understand HCG-based devices. The feature of beam shaping of the finite-size HCG was experimentally confirmed recently [18].

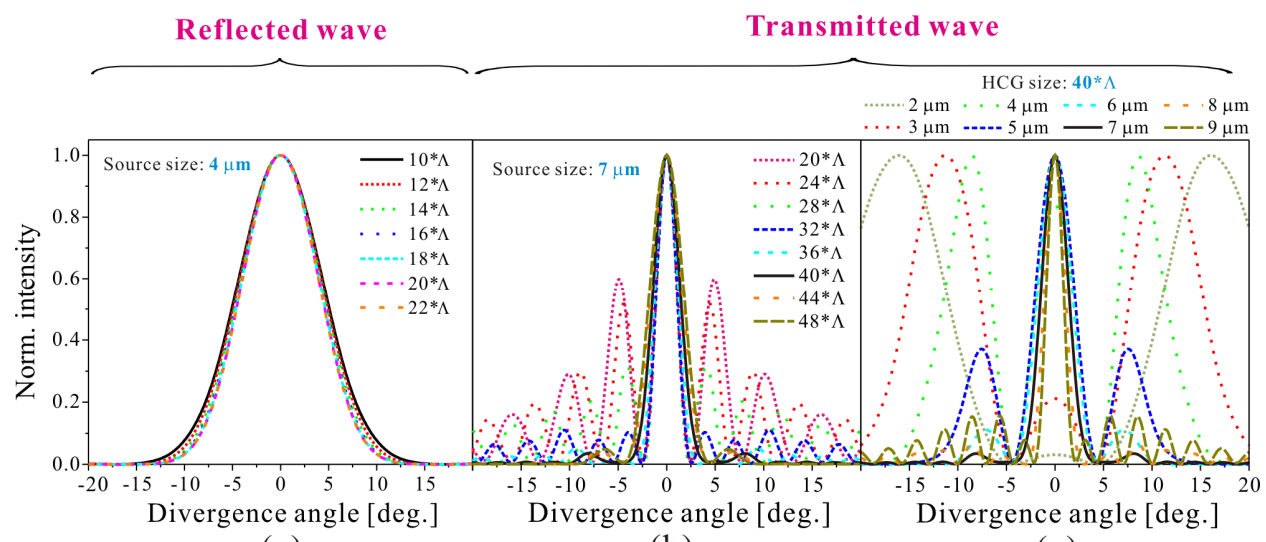

(a)

(b)

(c)

Fig. 4. (a) Far field profiles of the reflected waves ( $H_{y}$ component) under different HCG sizes at a wavelength of $849.54 \mathrm{~nm}$. (b) Far field profiles of the transmitted waves ( $H_{y}$ component) through the finite-size HCG at a wavelength of $849.54 \mathrm{~nm}$ for different HCG sizes. (c) Far field profiles of the transmitted waves ( $H_{y}$ component) through the finite-size HCG at a wavelength of $849.54 \mathrm{~nm}$ for different source sizes (1/e width of the Gaussian source) with the HCG size of $15.2 \mu \mathrm{m}$ $(40 * \Lambda)$. HCG parameters: $\Lambda=0.38 \mu \mathrm{m}, t=0.235 \mu \mathrm{m}, a=0.25 \mu \mathrm{m}$.

\section{UNIDIRECTIONAL TRANSMISSION IN A FINITE-SIZE HCG}

There are lots of higher-order angular components in the finite-size incident wave, for example, a finite-size Gaussian wave. When the finite-size Gaussian wave impinges on the HCG section as shown in Fig. 1, the incident wave excites the eigenmodes of the HCG. The eigenmodes of the HCG reduce the reflection and increase the transmission. At the same time, the eigenmode can redirect the vertically (in the $z$ direction) incident wave into the in-plane direction (in the $x$ direction) to the slab at each side, as shown in Figs. 5(a) and (b). Eigenmode 1 in Fig. 2(b) has an even symmetry, while Eigenmode 2 in Fig. 2(c) has an odd symmetry with respect to the plane along the $x$ direction. Thus, Eigenmodes 1 and 2 can be coupled to TM0 and TM1 modes of the slab, respectively, as shown in 
Fig. 6. This coupling between the eigenmodes in the HCG section and the modes in the slabs is achieved under the conditions of energy conservation, momentum conservation, and symmetry conservation. However, when the incident wave is from the slab in the in-plane direction, the incident wave is reflected, as shown in Fig. 7. No incident wave can be redirected into the vertical direction (in the $z$ direction) from the in-plane direction (in the $x$ direction) [17].

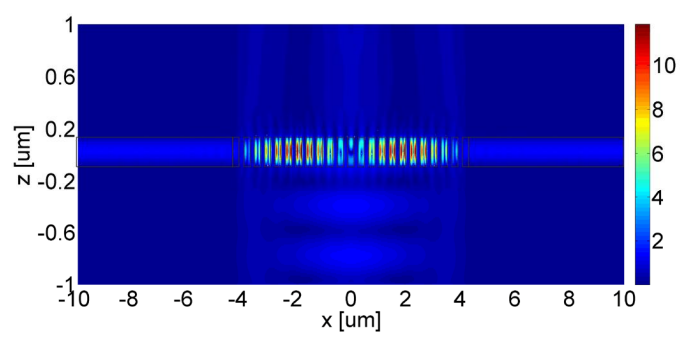

(a)

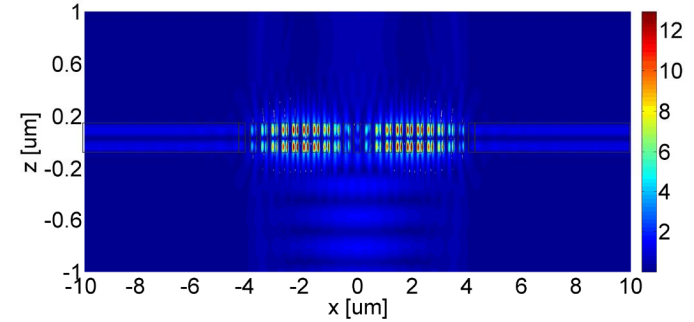

(b)

Fig. 5. (a) Field distribution of mode 1 ( $H_{y}$ component); (b) Field distribution of mode 2 ( $H_{y}$ component). The HCG parameters are: $\Lambda=0.38 \mu \mathrm{m}, t=0.235 \mu \mathrm{m}$, and $a=0.26 \mu \mathrm{m}$.

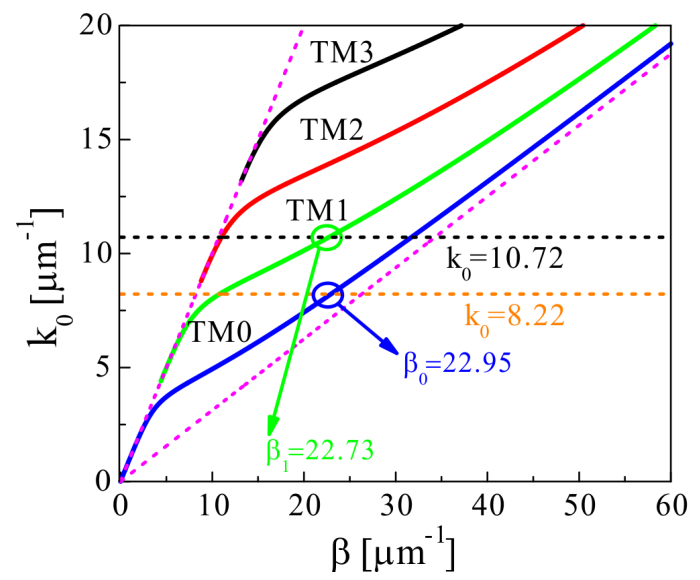

Fig. 6. Dispersion diagram for the slab for TM modes. The HCG parameters are: $\Lambda=0.38 \mu \mathrm{m}, t=0.235 \mu \mathrm{m}$, and $a=0.26 \mu \mathrm{m}$.

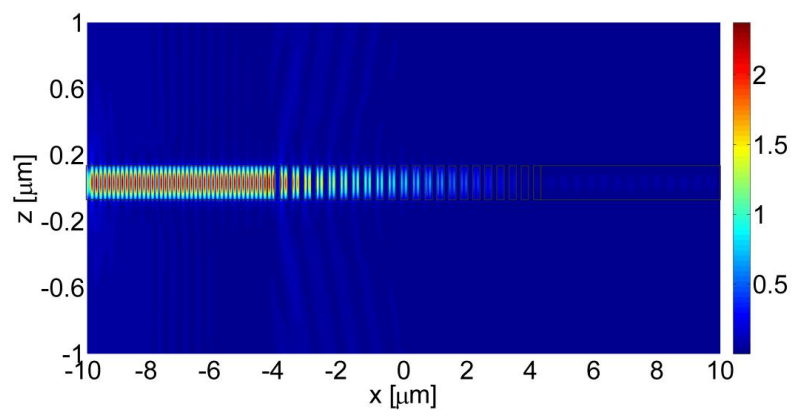

Fig. 7. Field distribution with the incident wave from the slab at the wavelength of $763.3 \mathrm{~nm}$. The HCG parameters are: $\Lambda=0.38 \mu \mathrm{m}, t=0.235 \mu \mathrm{m}$, and $a=0.26 \mu \mathrm{m}$.

\section{MONOLITHICALLY INTEGRATED HCG-BASED SENSOR}

As shown in Figs. 5(a) and (b), higher-order angular components in the finite-size incident wave can excite the eigenmodes in the finite-size HCG. The excited eigenmodes in the finite-size HCG have two features: (a) they are low- $Q$ guided modes; (b) they can transform part of the normally finite-size incident wave into the in-plane direction. Therefore, the guided mode should be very sensitive to parameter variations of the HCG, which can be exploited for optical sensors to detect the presence or the amount of biochemicals and chemicals. On the other hand, different from the detection schemes commonly adopted in the current optical sensing technologies, the optical detection unit can be easily 
integrated on-chip together with the HCG, benefiting from the redirected light. Thus an integrated HCG-based optical sensor for biochemicals and chemicals is proposed with the even guided mode, as shown in Fig. 8 [16]. The VCSEL is monolithically integrated with the HCG layer and the $p-i-n$ photodiode is also integrated in the same HCG layer. The HCG ensures polarization-stable operation of the VCSEL which is critical for the optical sensor. Note that the integrated optical sensor can be easily extended to $1 \mathrm{D}$ and $2 \mathrm{D}$ arrays which can greatly improve the throughput, and different chemicals can be detected simultaneously within the array [19].

For the sensing measurement, the same method is used as for optical sensors in [20]. At a fixed temperature, one current corresponds to one wavelength for a single-mode VCSEL. Therefore, the detected current at the integrated $p-i-n$ photodiode versus the bias current of the VCSEL can be mapped directly. The maximum current of the $p-i-n$ photodiode corresponds to a given current, and the wavelength of the guided mode in the finite-size HCG can be obtained. For the bulk sensing, a considerable portion of the guided mode is in the air region, out of the HCG bars. Thus, when the analytes or different concentrations of the same analyte (such as chemicals, DNA, protein, and volatile compounds) in bulk liquid or gaseous phase are present in the air region, the wavelength shift of the guided mode occurs without detection specificity. When the refractive index (i.e. $n_{\mathrm{L}}$ ) of the analyte increases the wavelength of the guided mode shifts to the longer wavelength. According to the wavelength shift of the guided mode the sensitivity is $287.66 \mathrm{~nm} / \mathrm{RIU}$ by the linear fitting, as shown in Fig. 9. This sensitivity is $>2$ times as large as that of the guide-mode resonance gratingbased optical sensor in [20].

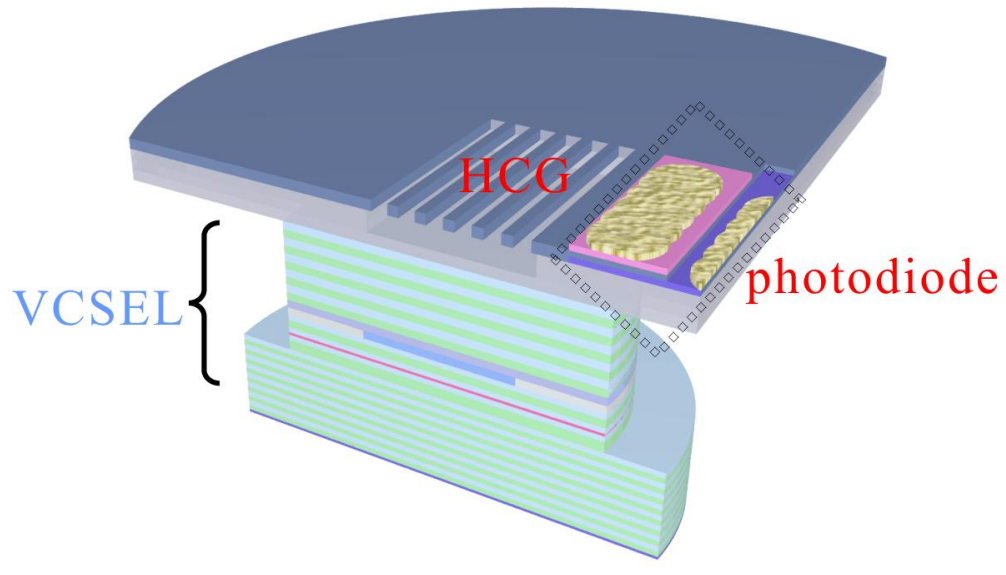

Fig. 8. Schematic of a monolithically integrated HCG-based optical sensor.

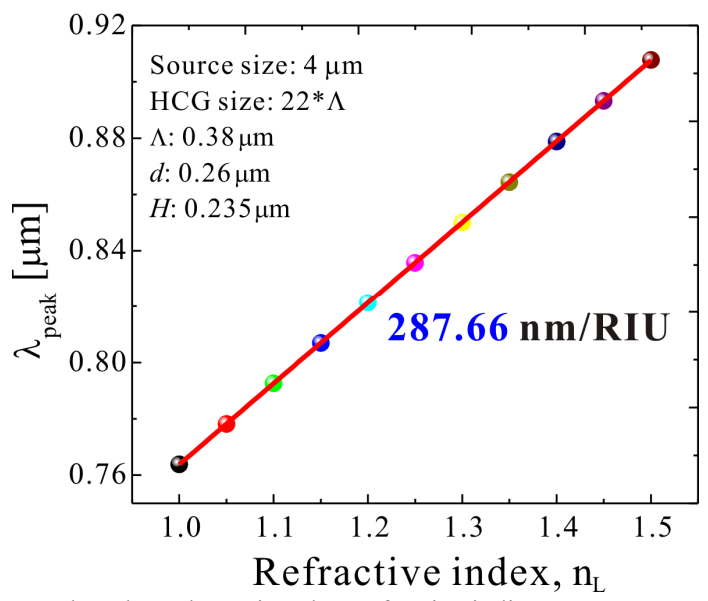

Fig. 9. Linear dependence of the peak wavelength on the various low refractive indices. 


\section{INTEGRABLE GAAS-BASED HCG REFLECTOR}

Integrable GaAs-based HCGs with $\mathrm{Ga}_{0.51} \mathrm{In}_{0.49} \mathrm{P}$ as the sacrificial layer were designed, fabricated, and characterized, targeting applications for high-speed VCSELs and its array in the wavelength range of larger than $900 \mathrm{~nm}$ [21]. In contrast to $\mathrm{GaAs}(\mathrm{AlInP})$ and $\mathrm{GaAs}(\mathrm{AlGaAs})$ material systems for GaAs-based HCGs which contain $\mathrm{Al}$ in the sacrificial layers, we used $\mathrm{Ga}_{0.51} \mathrm{In}_{0.49} \mathrm{P}$, which is lattice matched to GaAs and also has a high etching selectivity to GaAs in the $\mathrm{HCl}$ etching solution, to serve as the sacrificial layer to avoid the problem of oxidation of the remaining sacrificial layer [22]. As shown in Fig. 10(a), the HCGs are fabricated on a wafer that is composed of a 362-nm GaAs layer, a 1- $\mu \mathrm{m}$ $\mathrm{Ga}_{0.51} \mathrm{In}_{0.49} \mathrm{P}$ sacrificial layer, and a GaAs substrate. The period of the HCG is $600 \mathrm{~nm}$, and the width of the bar is varied with a step size of $10 \mathrm{~nm}$ around a central width of $390 \mathrm{~nm}$. The scanning electron microscope image of the fabricated HCG after selective etching and grating release is shown in Fig. 10(b). Figure 10(c) shows the calculated and measured reflectivity spectra of the HCG with a bar width of $410 \mathrm{~nm}$. The measured reflectivity spectrum is consistent well with the calculated reflectivity spectrum. For comparison, the reflectivity of the GaAs surface without the HCG pattern is also measured, which can clearly show the enhanced reflectivity after the GaAs surface is patterned with the HCG.

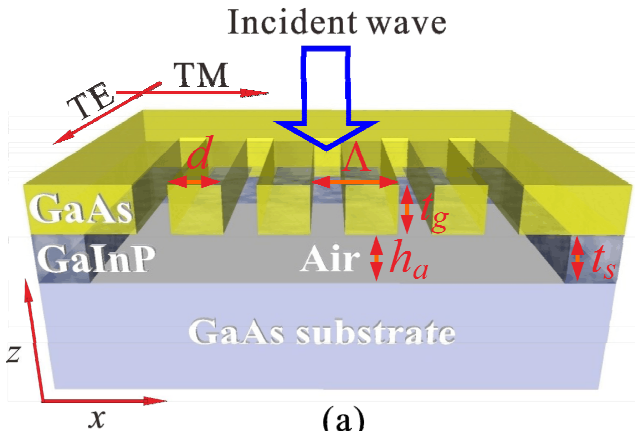

(a)

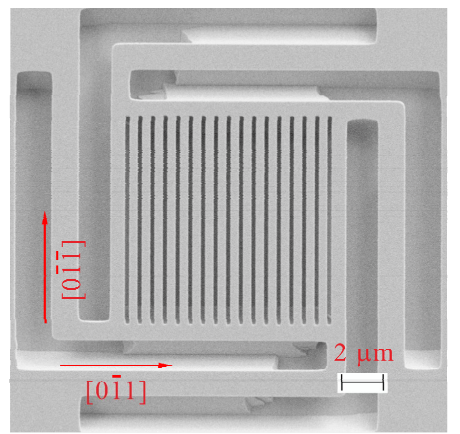

(b)

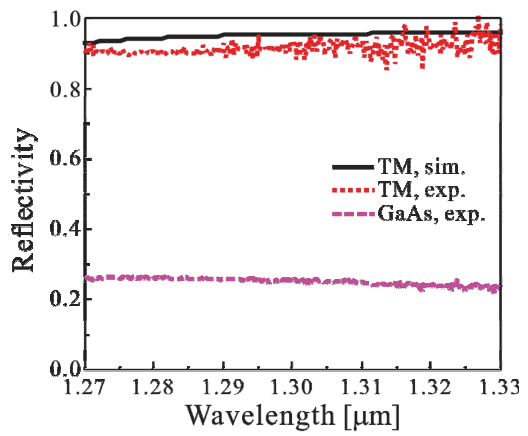

(c)

Fig. 10. (a) Schematic of the HCG. $\Lambda$ is the period of the HCG; $d$ is the width of the bars; $t_{g}$ is the thickness of the GaAs layer; $h_{a}$ is the thickness of the air gap after selective etching and grating release; $t_{s}$ the thickness of the $\mathrm{Ga}_{0.51} \mathrm{In}_{0.49} \mathrm{P}$ sacrificial layer. TE, transverse electric, the electric component is parallel to the HCG bars; TM, transverse magnetic, the electric component is perpendicular to the bars. The arrow denotes the incident wave. (b) Scanning electron microscope image of the fabricated HCG after selective etching and grating release. The arrows represent the crystal orientations. The size of the HCG is $10 \mu \mathrm{m} \times 10 \mu \mathrm{m}$. (c) Calculated and measured reflectivity spectra of the HCG with a bar width of $410 \mathrm{~nm}$.

\section{CONCLUSIONS}

Vertical-cavity surface-emitting lasers (VCSELs) are enablers for advanced data communication, sensing, and consumer electronics. Most important properties are their circular beam, low power consumption, low cost, large reliability, and the ability for 2D array configurations. Great efforts have been carried out in the past years to improve the performance of VCSELs. High-contrast metastructures like HCGs can be integrated to VCSELs to replace the complete top DBR or part of the DBR leading to novel VCSELs with new functionalities. A finite-size HCG exhibits completely different properties from an infinite-size HCG. A finite-size HCG exhibits completely different properties from an infinite-size HCG. This characteristic lets the finite-size HCG bridge a VCSEL and an in-plane photonic circuit for monolithically integrated sensors. The emerging optical properties of the finite-size HCG are expected to open new fields of applications of VCSELs and photonic integrated circuits.

\section{ACKNOWLEDGEMENTS}

A. Liu was supported by the National Natural Science Foundation of China (61675193), the Chinese Academy of Sciences (CAS) Pioneer Hundred Talents Program, and the Alexander von Humboldt Postdoctoral Fellowship. A. Liu and W. Zheng were partly supported by the Chinese National Key Basic Research Special Fund (2017YFA0206401), and the Strategic Priority Research Program of the Chinese Academy of Sciences (XDB24020100). D. Bimberg gratefully acknowledges a "Distinguished Fellowship" of the president of the Chinese Academy of Sciences. 


\section{REFERENCES}

[1] F. Koyama, "Recent advances of VCSEL photonics," J. Lightwave Technol. 24, 4502-4513 (2006).

[2] H. Li, P. Wolf, P. Moser, G. Larisch, J. A. Lott, and D. Bimberg, "Vertical-cavity surface-emitting lasers for optical interconnects," SPIE Newsroom, 25 Nov. 2014.

[3] A. Larsson, “Advances in VCSELs for communication and sensing," J. Sel. Top. Quantum Electron. 17, 1552-1567 (2011).

[4] R. Michalzik, VCSELs - Fundamentals, Technology and Applications of Vertical-Cavity Surface-Emitting Lasers, Springer Series in Optical Sciences, 166 (Springer, 2013).

[5] G. Larisch, P. Moser, J. A. Lott, and D. Bimberg, "Impact of photon lifetime on the temperature stability of $50 \mathrm{~Gb} / \mathrm{s} 980 \mathrm{~nm}$ VCSELs," IEEE Photon. Technol. Lett. 28, 2327-2330 (2016).

[6] P. Wolf, H. Li, A. Caliman, A. Mereuta, V. Iakovlev, A. Sirbu, E. Kapon, and D. Bimberg, "Spectral efficiency and energy efficiency of pulseamplitude modulation using 1.3 $\mu \mathrm{m}$ wafer-fusion VCSELs for optical interconnects," ACS Photonics 4, 2018-2024 (2017).

[7] A. Liu, and D. Bimberg, "Vertical-cavity surface-emitting lasers with nanostructures for optical interconnects," Front. Optoelectron. 9, 249-258 (2016).

[8] V. Karagodsky, F. G. Sedgwick, and C. J. Chang-Hasnain, "Theoretical analysis of subwavelength high contrast grating reflectors," Opt. Express 18, 16973-16988 (2010).

[9] W. Zhou, D. Zhao, Y.-C. Shuai, H. Yang, S. Chuwongin, A. Chadha, J.-H. Seo, K. X. Wang, V. Liu, Z. Ma, and S. Fan, "Progress in 2D photonic crystal Fano resonance photonics," Progress in Quantum Electronics 38, 1-74 (2014).

[10] R. Magnusson and M. Shokooh-Saremi, "Physical basis for wideband resonant reflectors," Opt. Express 16, 3456-3462 (2008).

[11] M. C. Y. Huang, Y. Zhou, and C. J. Chang-Hasnain, "A surface-emitting laser incorporating a high-index-contrast subwavelength grating,” Nat. Photonics 1, 119-122 (2007).

[12] S. Boutami, B. Benbakir, J.-L. Leclercq, and P. Viktorovitch, "Compact and polarization controlled $1.55 \mu \mathrm{m}$ vertical-cavity surface emitting laser using single-layer photonic crystal mirror," Appl. Phys. Lett. 91, 071105 (2007).

[13] A. Liu, F. Fu, Y. Wang, B. Jiang, and W. Zheng, "Polarization-insensitive subwavelength grating reflector based on a semiconductor-insulatormetal structure," Opt. Express 20, 14992-15000 (2012).

[14] A. Liu, W. Hofmann, and D. Bimberg, "Two dimensional analysis of finite size high-contrast gratings for applications in VCSELs," Opt. Express 22, 11804-11811 (2014).

[15] A. Liu, W. Zheng, and D. Bimberg, "Comparison between high- and zero-contrast gratings as VCSEL mirrors," Opt. Commun. 389, 35-41 (2017).

[16] A. Liu, W. Hofmann, and D. Bimberg, "Integrated high-contrast-grating optical sensor using guided mode," IEEE J. Quantum Electron. 51, $6600108(2015)$.

[17] A. Liu, W. Zheng, and D. Bimberg, "Unidirectional transmission in finite-size high-contrast gratings," Asia Communications and Photonics Conference, paper AF2A.52 (2016).

[18] K. Li, Y. Rao, C. Chase, W. Yang, and C. J. Chang-Hasnain, "Monolithic high-contrast metastructure for beam-shaping VCSELs," Optica 5, 10$13(2018)$.

[19] A. Liu, W. Hofmann, and D. Bimberg, "Optical sensor for detecting chemical, biochemical or biological substances," US Patent, Patent No. 9693715 (2017).

[20] C. F. R. Mateus, M. C. Y. Huang, P. Li, B. T. Cunningham, and C. J. Chang-Hasnain, "Compact label-free biosensor using VCSEL-based measurement system," IEEE Photon. Technol. Lett. 16, 1712-1714 (2004).

[21] A. Liu, P. Wolf, J.-H. Schulze, and D. Bimberg, "Fabrication and characterization of integrable GaAs-based high-contrast grating reflector and Fabry-Pérot filter array with GaInP sacrificial layer," IEEE Photonics J. 8, 2700509 (2016).

[22] E. Weidner, S. Combrié, N.-V.-Q. Tran, A. D. Rossi, J. Nagle, and S. Cassette, A. Talneau, and H. Benisty, "Achievement of ultrahigh quality factors in GaAs photonic crystal membrane nanocavity," Appl. Phys. Lett. 89, 221104 (2006). 